\title{
The ATLAS High Level Trigger Steering Framework and the Trigger Configuration System.
}

\author{
Tiago Pérez Cavalcanti ${ }^{1}$ \\ DESY \\ Notkestrasse 85, Hamburg, Germany \\ E-mail: tiago.perezedesy.de

\section{On behalf of the ATLAS TDAQ Collaboration ${ }^{2}$}

The ATLAS experiment, installed in the Large Hadron Collider (LHC) at CERN, is designed to study proton-proton and nucleus-nucleus collisions with a maximum centre of mass energy of $14 \mathrm{TeV}$ at a bunch collision rate of $40 \mathrm{MHz}$. In March 2010 the four LHC experiments saw the first proton-proton collisions at $7 \mathrm{TeV}$. Already within the first year, a collision rate of nearly $10 \mathrm{MHz}$ was achieved.

Events of potential interest are selected by a three-level trigger system, with a final recording rate of about $200 \mathrm{~Hz}$. The first level (L1) is implemented in custom hardware; the two levels of the high level trigger (HLT) are software triggers, running on large computing farms. Within the ATLAS physics program more than 500 trigger signatures are defined. The HLT tests each signature on each event accepted by the L1. The HLT Steering is the software framework, which runs and controls the processing of the high level triggers. It ensures that each signature is tested independently to guarantee unbiased decisions. In order to minimise data transfer and execution time, the HLT Steering caches and reuses results of algorithms shared among several triggers. Some signature tests are performed only on a scaled-down fraction of candidate events, this reduces the output rate and limits execution time.

The trigger configuration system dynamically sets up the online L1 and HLT systems. It also archives the trigger configuration for offline analysis. The configuration system uses a relational database (TriggerDB) to store all configuration information. A graphical user interface, the Trigger Tool, has been developed to interact with the TriggerDB.

This work will introduce the ATLAS trigger with focus on the trigger configuration, steering and resource monitoring system. Performance figures from the first year of successful operation will be presented.

XLIX International Winter Meeting on Nuclear Physics- BORMIO2011

Bormio, Italy

January 24-28 2011

Speaker

ATL-DAQ-PUB-2010-002, Geneva, 2010 [http: //cdsweb.cern.ch/record/1265604 ] 


\section{Introduction}

ATLAS [1] (Figure 1) is a general-purpose particle detector experiment installed at the CERN Large Hadron Collider (LHC). It was designed to study proton-proton and heavy ion collisions at a maximum rate of $40 \mathrm{MHz}$. ATLAS is a complex experiment, which features seven main sub-detectors divided into 3 detector layers. Moving from the interaction point outwards, these are:

\section{The Inner Detector}

This layer combines the information from the Pixel Detector, the Semiconductor Tracker (SCT) and the Transition Radiation Tracker (TRT). The main tasks of these detectors are to provide tracking close to the interaction point, and to reconstruct the position of the primary vertex. They also provide momentum measurement and charged particle identification [1].

\section{Calorimeters}

The Liquid Argon (LAr) and the Scintillating Tile calorimeters provide the measurement of the total energy carried by the hadrons and leptons produced in the collisions.

\section{Muon Spectrometer}

The third and outermost layer is devoted to muon identification and reconstruction. Two fast detectors, the Resistive Plate Chambers (RPC) and the Thin Gap Chambers are used mainly to deliver trigger information. The Monitored Drift Chambers (MDT) data are used to perform tracking and reconstruction of muon momenta.

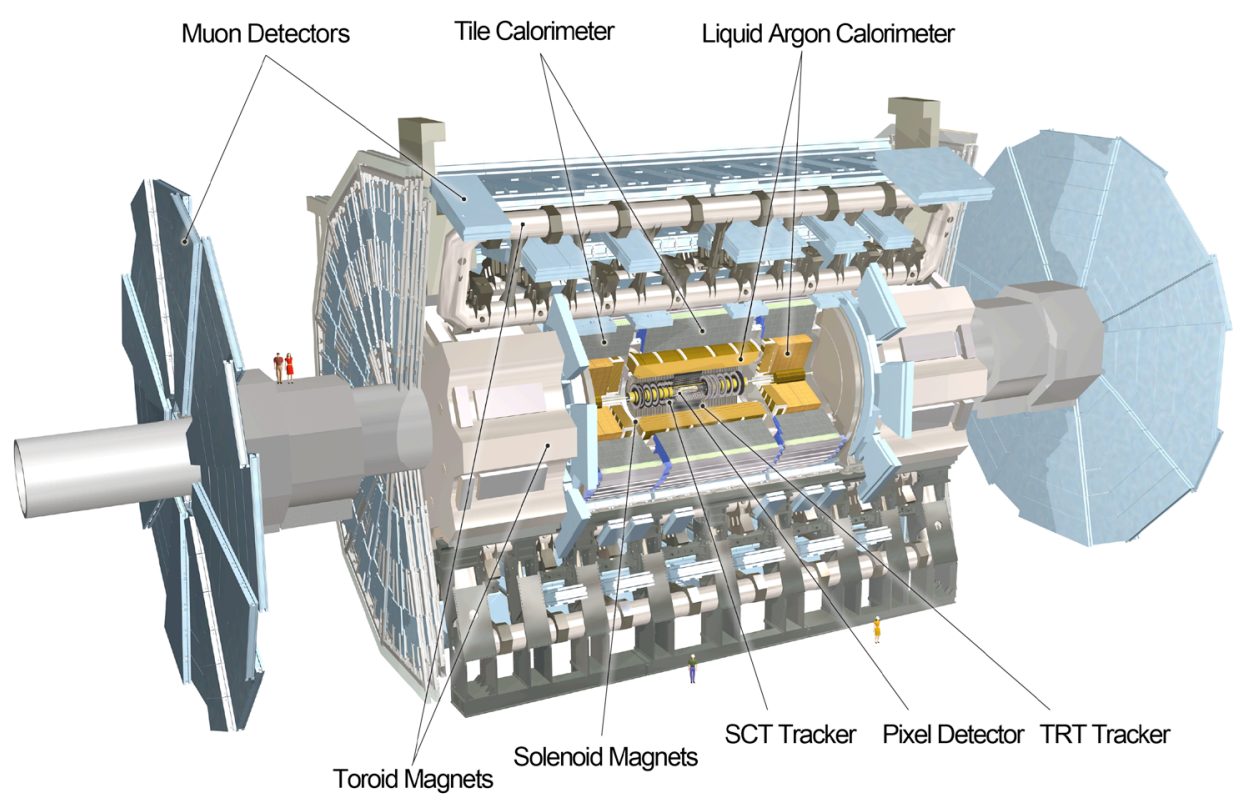

Figure 1 ATLAS experiment at LHC. The people on the picture are drawn to scale to provide a reference of the size of the detector. 
In March 2010 the four LHC experiments saw the first proton-proton collisions at $7 \mathrm{TeV}$ centre of mass energy. At the end of the proton-proton run in October 2010, a collision rate of nearly $10 \mathrm{MHz}$ was achieved. During this run ATLAS featured a recording efficiency of up to $93.6 \%$ and obtained a data sample of $45 \mathrm{pb}^{-1}$. During the 3 weeks of heavy ion running in December 2010, the luminosity recorded was $9.1 \mu \mathrm{b}^{-1}[2]$.

\section{The ATLAS Trigger System}

For proton-proton collisions at $7 \mathrm{TeV}$, cross sections for precision measurements like the leptonic $\mathrm{W}$ decay are of the order of $10^{7}$ times smaller than the total cross section. For interesting new physics processes like Higgs production, the cross section is even $10^{10}$ times smaller (Figure 2)[3]. To enhance the content of events of potential interest in the recorded data, a three level trigger system was developed. At ATLAS, collisions of potential interest for physics are selected by the trigger system. The ATLAS trigger filters in real time all collisions detected to reduce the event rate from the several $\mathrm{MHz}$ collision rate to a recording rate of about $200 \mathrm{~Hz}$.

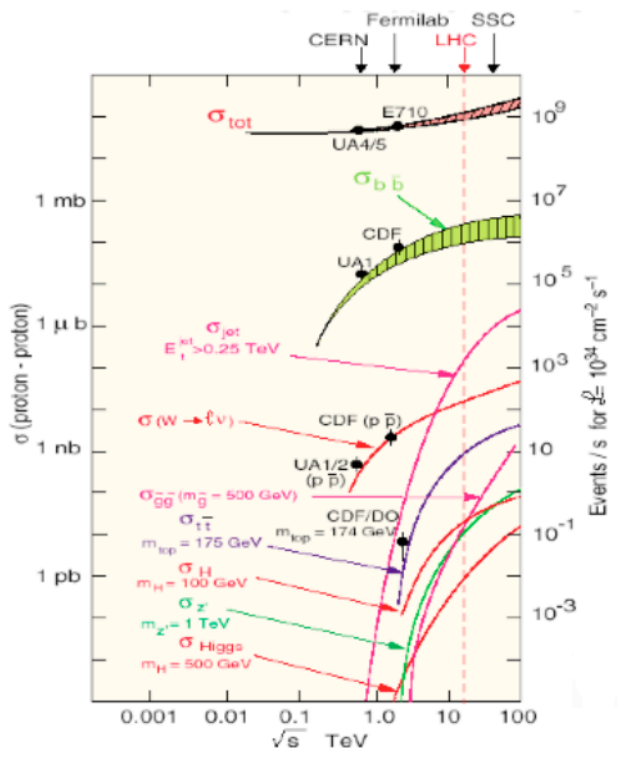

Figure 2 Cross section of different processes as a function of centre of mass energy [3].

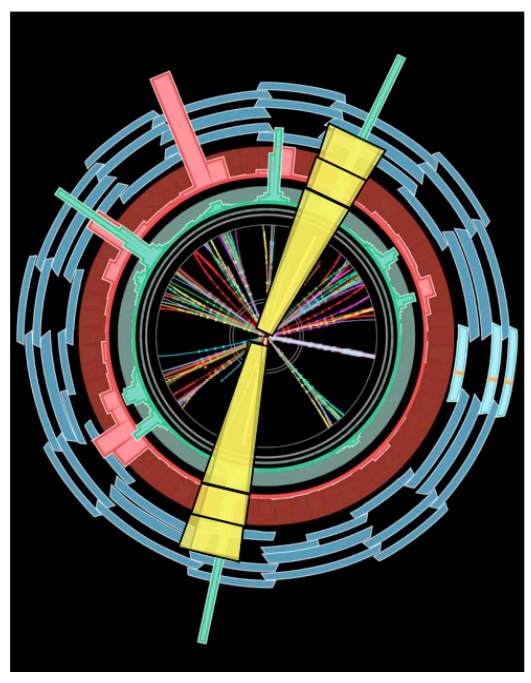

Figure 3 Simulated jet event showing the regions of interest, RoI, in yellow.

A schematic diagram of the data flow in the ATLAS Trigger and Data Acquisition System is depicted in Figure 4. The green arrows illustrate the main data path from the detector to permanent storage. As the diagram shows, trigger levels 1 and 2 draw only the portions of data they need to produce a trigger decision. The Event Filter gets entire events from the event builder, thus it has the complete event information available for processing. The numbers on the figure are the approximate values for the $7 \mathrm{TeV}$ proton-proton run during 2010 [4]. 


\subsection{Level 1 Trigger}

The level 1 trigger (L1) is implemented in custom hardware. Detector specific modules analyse data from the calorimeter, muon and minimum bias detectors and forward partial decision to the central trigger processor (CTP). There, the partial decisions are combined and a final L1 decision is taken. If an event is accepted, detectors are read out and data are buffered by the read-out system (ROS). In ATLAS, the trigger analyses events looking for trigger signatures in a segmented manner. These segments, called regions of interest or RoI's (Figure 3), could be visualized, in the barrel part, as a cone expanding from the interaction point outwards. Whenever an event is positively triggered, the location of the interesting features, i.e. the RoI together with the trigger information is forwarded to the Level 2 trigger system.

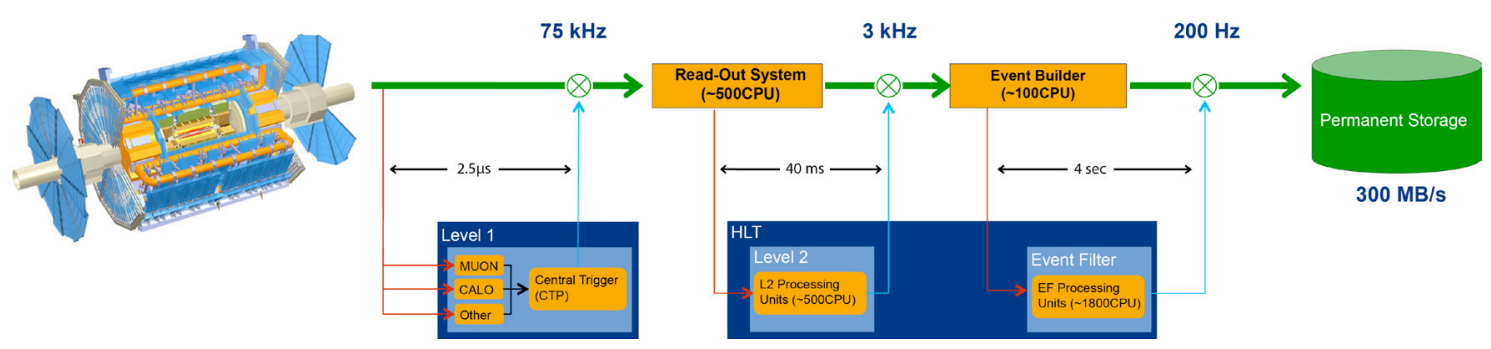

Figure 4 Schematic representation of the ATLAS Trigger System. The green arrows show the main data path from the detector to permanent storage. Numbers depicted are the approximate values for the $7 \mathrm{TeV}$ proton-proton run during 2010 [4].

\subsection{The High Level Trigger}

The two levels of the high level trigger (HLT), known as Level 2 (L2) and Event Filter $(\mathrm{EF})$, are software triggers running on large farms of standard computers and network devices.

The Level 2 trigger performs a partial reconstruction of selected events based on the information of the location of interesting features (RoI) passed on by the Level 1. Events accepted by the Level 2 are assembled by the Event Builder and forwarded to the third trigger layer, the Event Filter. The Event Filter reconstructs events with full detector resolution in the regions selected by L2, using the same tools as those used during offline reconstruction and draws a final trigger decision. The event filter takes a decision in a short time; about 1 second for proton-proton collisions and about 4 seconds for heavy ion runs.

\section{The Trigger Configuration System}

The trigger configuration system [5](Figure 5) is the system that controls the functioning of the trigger. It stores trigger parameters and provides a way to interact with the trigger system. Both online and offline operations are foreseen, and therefore tools to review and modify trigger 
parameters during data taking, as well as to query offline trigger configuration for specific runs, have been developed.

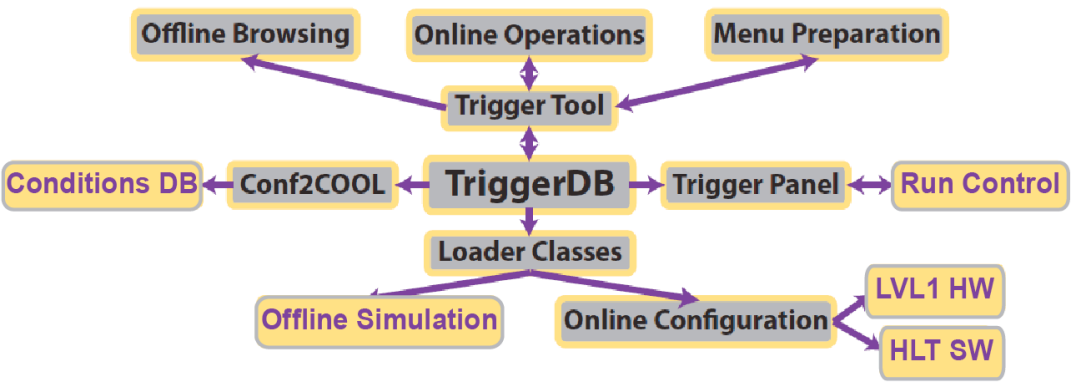

Figure 5 The trigger configuration system. In the centre, the Trigger DB holds all information used by the ATLAS trigger system [6]. In yellow, external systems interfacing with the configuration system.

\subsection{The Trigger DB}

The central part of the configuration system is the trigger database. The trigger DB is realized as a relational database. It holds the trigger menu and provides concurrent access to the trigger parameters to all tools in the configuration system. Figure 6 shows a simplified snapshot of the trigger database schema, where only the top-level tables are drawn.

The trigger menu is the realization of the ATLAS trigger program. In a simplistic picture the trigger menu would be a list of trigger signatures (Figure 7), selection algorithms and prescaling values. In ATLAS, a trigger signature is each of the features analysed by the trigger to yield a trigger decision; these could be identifying particle candidates (i.e. leptons, jets etc.) or determining event properties (i.e. total energy, missing transverse energy etc.). Figure 7 shows how the trigger signature is implemented in a chain of Level 1, Level 2 and Event Filter to select a muon with more than $6 \mathrm{GeV}$ transverse momentum (MU6). As shown on the figure, a signature spans all three trigger levels. At each level, a signature has reconstruction and selection algorithms, which are executed in order.

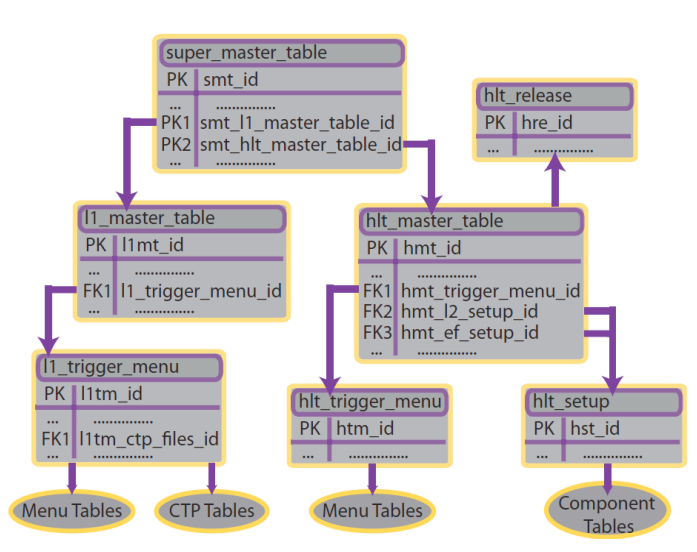

Figure 6 Simplified schema of the Trigger DB.

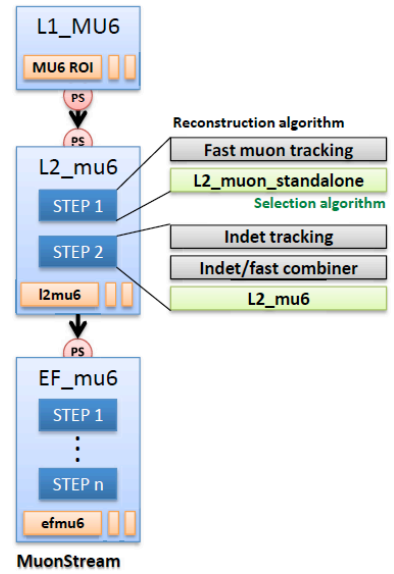

Figure 7 Schematic view of a trigger signature. 
Each signature at each level has an associated prescale factor (PS in Figure 7), which can be used to control the rate of a specific signature, or to disable it completely. The prescale value represents the number of rejected events before an event is let through; hence the final rate would be equal to the algorithms accepting rate over the prescale value. Thus a value of 1 means that the final rate is equal to the signature rate i.e. there is no prescale, and values towards infinity will lead to a zero accepted rate. By convention, prescale values 0 or negative disable the signature. To save computing resources in the HLT triggers, prescales are applied before computing the signature.

\subsection{The Trigger Tool}

The Trigger Tool is a JAVA application, which interacts with the trigger database, and hence with the whole trigger configuration system. It provides a graphical user interface (see Figure 8) to conveniently edit and review the trigger menu interactively. Users can access all parameters of a menu using the Trigger Tool, thus it provides a graphical and convenient manner to perform all trigger operations. The Trigger Tool also has support for command line interaction. This allows for automation of repetitive actions, and provides trigger interaction in scripts.

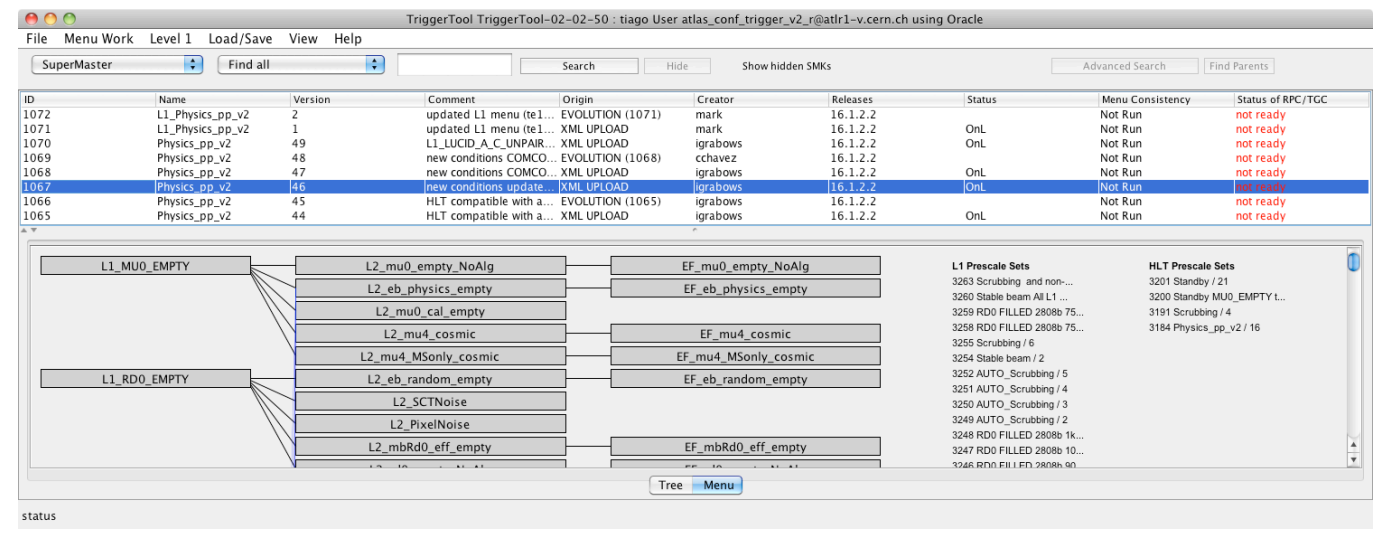

Figure 8 The Trigger Tool main panel. The upper frame displays the list of trigger menus available. The lower frame the tree of trigger chains for the selected menu.

The Trigger Tool also features an authentication layer with several levels of user access rights to the trigger DB. There are 3 main user levels recognised by the Trigger Tool: user, shifter and expert. The level user provides read only access to the trigger configuration. This is very useful for general ATLAS members to quickly review trigger settings. The trigger operator at the ATLAS control room uses the shifter level. This provides edit capabilities to those trigger elements, which may need modification during data taking, i.e. changing prescale values, enabling/disabling chains, etc. Trigger experts will normally use the expert mode. In this mode the user has full editing capabilities to the complete trigger menu.

Complementary to the Trigger Tool, a web interface, the Trigger Configuration Browser, which provides a simple read only access to the trigger settings is also available. 


\subsection{HLT Steering}

The software framework, which runs, controls and monitors the high-level trigger, is called HLT Steering. The Steering receives the running information from the trigger configuration System. The HLT Steering works in a similar way for the two levels of high-level trigger, Level 2 and EF. The first step is to activate the signatures accepted in the previous level. The Steering will then process all active signatures until they can be rejected for any reason. If all signatures in one level are rejected, the event is then rejected. But if at least one is positive, then the event will be passed on to the next level, from Level 2 to EF, or from EF to offline storage.

At each step algorithms will be run to perform reconstruction and to test a hypothesis. The hypothesis tested may include, for example, applying a momentum cut or testing a matching criterion between two objects.

The Steering features an intelligent caching logic, which avoids running any algorithm twice. It is very common that many HLT signatures share algorithms and run on the same data set, in these situations the results of the first processing are reused.

\section{Trigger Operations}

\subsection{HLT Prescales Editing during Data Taking}

The ability to adjust HLT prescales, in addition to L1 prescales, during data taking was introduced before the 2010 run [7]. This is important since by tuning prescale values, it is possible to keep the output rate constant in spite of changes in the instantaneous luminosity (Figure 9). Besides that, it also gives the trigger operator the possibility to react to unforeseen situations like "hot spots" in the detector or over-active signatures blocking the HLT farm.

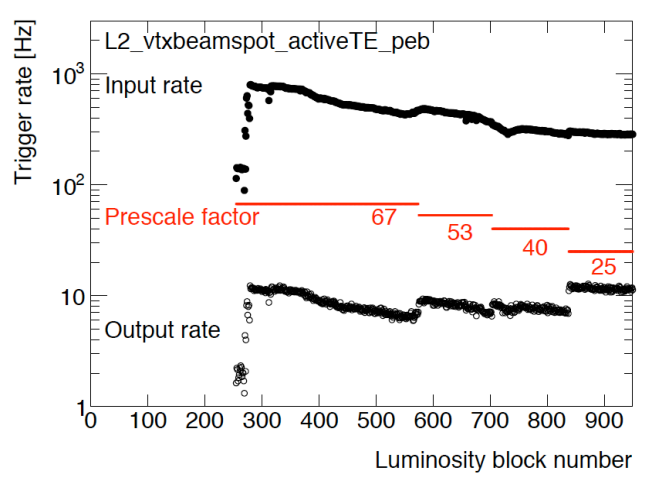

Figure 9 Tuning of the L2 output rate of an arbitrary algorithm by adjusting prescale factors.

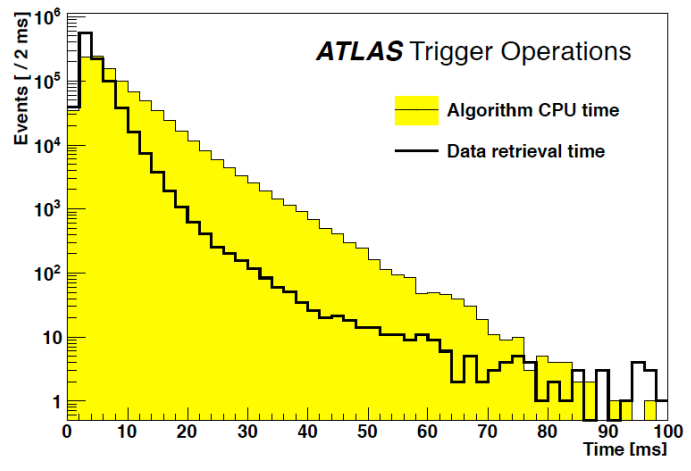

Figure 10 Example of resource monitoring in HLT for an arbitrary algorithm and run.

Records of which prescales are used for each signature and when they were changed are logged to the conditions database and are available for offline analysis. 


\subsection{Monitoring Performance and Resource Utilisation.}

The HLT Steering, as responsible for the execution of the HLT, also monitors the trigger rates and the resources utilized by the trigger signatures (Figure 10). To do so, the Steering collects information about the number of events passing each step of each trigger signature. This information is read out every 10 seconds for L2 and 20 seconds for EF, using such a slow sampling ratio keeps the overhead cost for monitoring small. In addition, performance histograms are collected by a hierarchical gathering infrastructure and passed to the online monitoring system for displaying.

\section{Conclusions}

The ATLAS Trigger has proven to be a reliable and well-performing system during the proton-proton and heavy ion runs in 2010. In the $7 \mathrm{TeV}$ proton-proton run, from March until October, ATLAS recorded $45 \mathrm{pb}^{-1}$ with a data recording efficiency of $93.6 \%$ [2]. During the 3 weeks of heavy ions run in December 2010, $9.1 \mu \mathrm{b}^{-1}$ were recorded. In both runs, the trigger configuration proved to be a flexible system, which allowed efficient reactions to all vicissitudes that appeared during data taking. The ability to easily modify prescales (L1 and HLT) on the fly was of great help in the early stages of constantly rising luminosities, as well as during stable operations, to optimize data taking. Beside that, the trigger rate and resource utilisation monitoring was an asset helping the trigger operators to maximise the data taking under all circumstances.

\section{References}

[1] The ATLAS Collaboration, The ATLAS Experiment at the CERN Large Hadron Collider, JINST 3 S08003 [doi: 10.1088/1748-0221/3/08/S08003]

[2] J. Stelzer, The ATLAS High Level Trigger Configuration and Steering, Experience with the First 7 TeV Collisions, in proceedings of CHEP 2010, Taipei 2010.

[3] C. Borer, Overview of the ATLAS data acquisition system operating at the TeV scale, in proceedings of $17^{\text {th }}$ IEEE NPSS Real Time Conference, Lisbon 2010. [ ATL-COM-DAQ-2010-067]

[4] J. Baines, Performance of the ATLAS Trigger with Proton Collisions at the LHC, in proceedings of $35^{\text {th }}$ ICHEP, Paris 2010.

[5] N. Berger et al, The ATLAS high level trigger steering. 2008 J. Phys.: Conf. Ser. 119022013 [doi:10.1088/1742-6596/119/2/022013]

[6] P. J. Bell, The Configuration System of the ATLAS Trigger, in proceedings of IEEE nuclear science and medical imaging conference, Orlando 2009.

[7] S. George, The ATLAS high level trigger configuration and steering software: Experience with 7 $\mathrm{TeV}$ collisions, in proceedings of $35^{\text {th }}$ ICHEP, Paris 2010. 